\title{
An Examination of the Consultation Component of a Training Protocol for Parent-Child Interaction Therapy
}

Carrie B. Jackson

Follow this and additional works at: https://researchrepository.wvu.edu/etd

\section{Recommended Citation}

Jackson, Carrie B., "An Examination of the Consultation Component of a Training Protocol for Parent-Child Interaction Therapy" (2017). Graduate Theses, Dissertations, and Problem Reports. 5869.

https://researchrepository.wvu.edu/etd/5869

This Thesis is protected by copyright and/or related rights. It has been brought to you by the The Research Repository @ WVU with permission from the rights-holder(s). You are free to use this Thesis in any way that is permitted by the copyright and related rights legislation that applies to your use. For other uses you must obtain permission from the rights-holder(s) directly, unless additional rights are indicated by a Creative Commons license in the record and/ or on the work itself. This Thesis has been accepted for inclusion in WVU Graduate Theses, Dissertations, and Problem Reports collection by an authorized administrator of The Research Repository @ WVU. For more information, please contact researchrepository@mail.wvu.edu. 
An Examination of the Consultation Component of a Training Protocol for Parent-Child Interaction Therapy

Carrie B. Jackson, B.S.

\author{
Thesis submitted \\ to the Eberly College of Arts and Sciences \\ at West Virginia University \\ in partial fulfillment of the requirements for the degree of \\ Master of Science in \\ Psychology
}

Amy D. Herschell, Ph.D., Chair

Cheryl B. McNeil, Ph.D.

Nicholas Turiano, Ph.D.

Department of Psychology

Morgantown, West Virginia

2016

Keywords: implementation, training, consultation, parent training

Copyright 2016 Carrie B. Jackson 


\begin{abstract}
An Examination of the Consultation Component of a Training Protocol for Parent-Child Interaction Therapy
\end{abstract}

Carrie Beth Jackson

There is a growing need to train clinicians in evidence-based treatments (EBTs) to increase the accessibility of research-informed treatments in community settings (Herschell, Kolko, Baumann, \& Davis, 2010). Multi-component trainings, which commonly include ongoing support, are more effective than one-day workshops, reading treatment manuals, and other brief training methods. The present study examines the effectiveness of one form of ongoing support, consultation, as part of a multi-component training protocol. Thirty-two community-based clinicians were trained in Parent-Child Interaction Therapy (PCIT) as part of a statewide implementation effort, and data were collected on several training (e.g., knowledge) and implementation (e.g., acceptability) outcomes at pre-, mid-, and post-training. Simple and multiple linear regression analyses were conducted to predict post-training knowledge, skill, acceptability, and feasibility, as well as to examine clinician variables that moderate these relations. Consultation call attendance significantly predicted post-training skill. However, the impact of consultation call attendance on skill was qualified by a significant interaction for PCIT caseload. Implications for training guidelines are discussed. 
Table of Contents

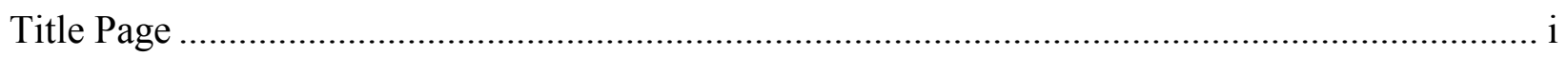



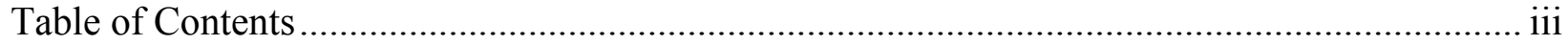

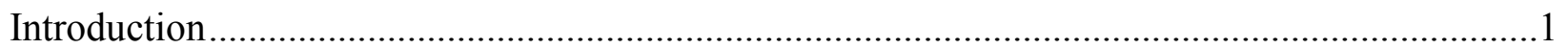

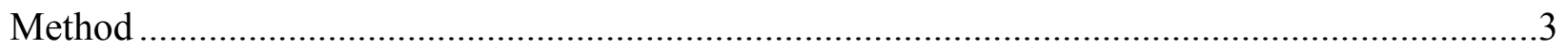



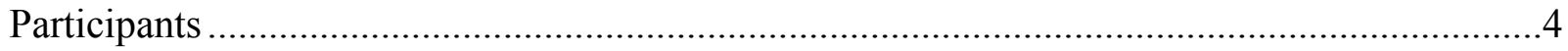

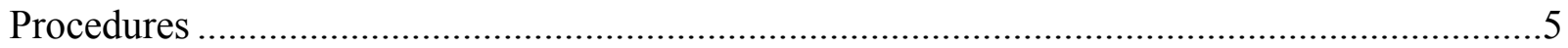

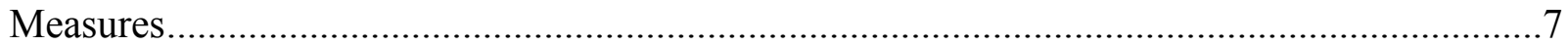

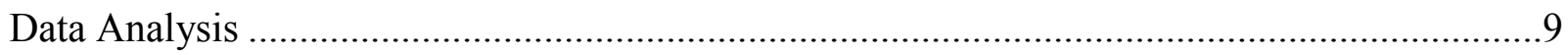

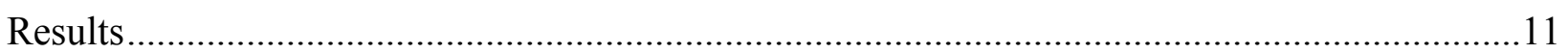

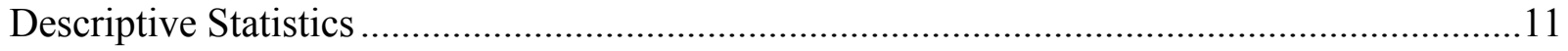

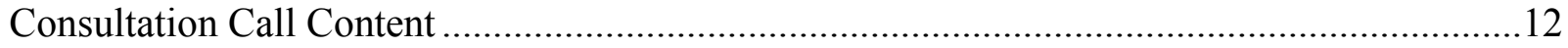

Consultation Call Attendance and Outcomes.................................................................... 12

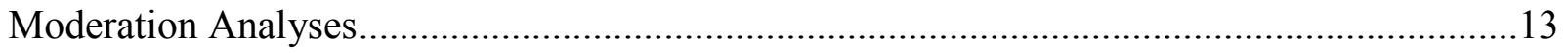

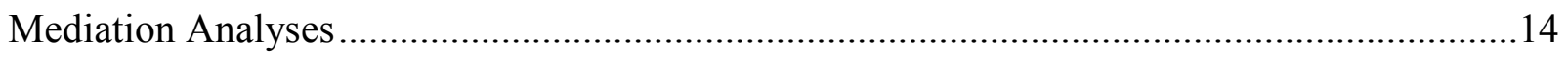

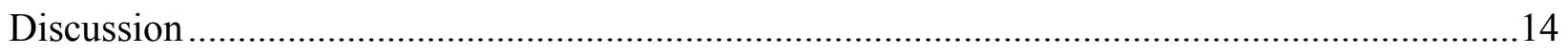

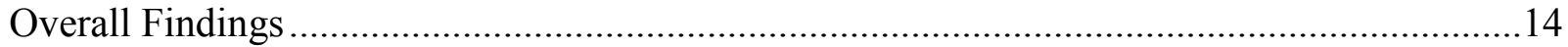





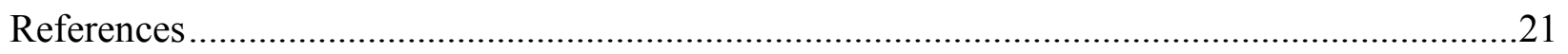

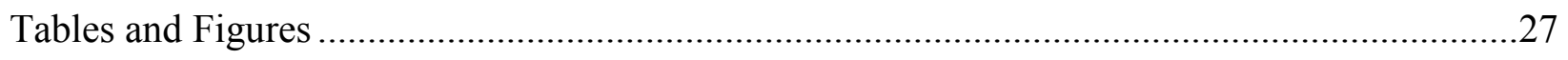

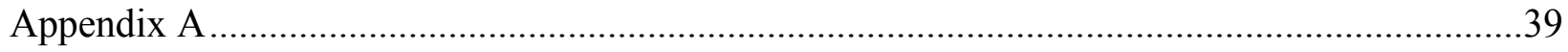

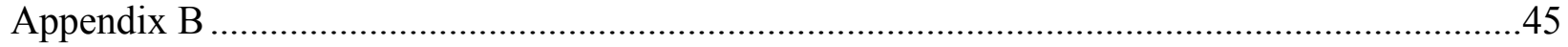

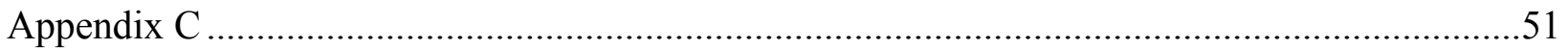


An Examination of the Consultation Component of a Training Protocol for

\section{Parent-Child Interaction Therapy}

Mental health disorders constitute a major public health concern in the United States, with an estimate of over four million children and adolescents suffering from a disorder that causes significant functional impairment (“Facts on Children's Mental Health,” 2010). Evidencebased treatments (EBTs) have been developed for a variety of behavior, mood, substance use, and developmental disorders; however, they are underutilized in community mental health settings where many individuals receive mental health services (Kataoka, Zhang, \& Wells, 2002; Silverman, Pina, \& Viswesvaran, 2008; Waldron \& Turner, 2008; Wampold et al., 2011; Wells \& Egan, 1988). Widening the gap between research and practice, many community-based clinicians have not received any formal training in EBTs (Rodríguez, Southam-Gerow, O'Connor, \& Allin, 2014; Weissman et al., 2006). At the same time, many state mental health systems have mandated clinician use of EBTs, while national and international initiatives have also called for the increased provision of these treatments (Glisson \& Schoenwald, 2005; Rieckman, Bergmann, \& Rasplica, 2013). This has resulted in the challenge of effectively training a large number of clinicians within a short amount of time.

Although there is a pressing need to train clinicians in EBTs, there is little agreement on the most effective way to do so (Jensen-Doss, Hawley, Lopez, \& Osterberg, 2009). Baldwin and Ford's training transfer model (see Baldwin \& Ford, 1988), provides a framework for understanding different factors that influence training outcomes. Three training input factors are hypothesized to influence training outcomes: (a) training design, such as training content and components, (b) individual trainee characteristics, including demographics and previous skills, and (c) work-environment factors, such as organizational climate and supervisory support. These 
input factors may have direct and indirect effects on training outcomes, by directly influencing immediate learning of training material, and also indirectly affecting the transfer of skills to the workplace (Baldwin \& Ford, 1988). Of particular relevance to the current paper are the training design and individual trainee characteristics input factors.

With regards to training design, research supports that individual training components such as workshops and treatment manuals are mostly ineffective for increasing clinician knowledge and skill (Herschell et al., 2010). It has been suggested that these training methods are too brief to allow clinicians to fully integrate newly learned skills into their current caseload (Beidas, Edmunds, Marcus, \& Kendall, 2012). A growing body of literature has recommended that multi-component trainings, which incorporate several training components, are the most effective strategy for training clinicians. Herschell and colleagues (2010) identified components routinely incorporated into multi-component trainings: (a) treatment manuals, (b) several days of intensive workshops, (c) expert consultation, (d) review of client sessions, (e) supervisor trainings, (f) booster trainings, and (g) completion of at least one training case. Ongoing support strategies, such as consultation, are often considered an essential aspect of the efficacy of multicomponent trainings (Beidas et al., 2012).

Consultation has been defined as communication between an expert in a field, the consultant, and a consultee who seeks advice from the consultant for improvement of a current work issue (Caplan, 1970). Research across several fields has suggested that consultation as part of training improves both provider and patient outcomes (Edmunds, Beidas, \& Kendall, 2013). Within the field of mental health, consultation call attendance has been linked to improvements in clinician knowledge, skill, and adherence as well as patient outcomes (Beidas et al., 2012; Schoenwald, Chapman, Sheidow, \& Carter, 2009). While these studies provide support for the 
importance of consultation, a majority of studies have examined the effects of training protocols as a whole, as opposed to dismantling training design factors which promote positive outcomes. Given this limitation, additional research is needed on the consultation component of training protocols.

Baldwin and Ford's (1988) training transfer model also suggests that individual trainee characteristics may influence training outcomes. Although research in other fields (e.g., industrial-organizational) has found evidence to support the influence of trainee characteristics on training outcomes (e.g., Velada, Caetano, Michel, Lyons, \& Kavanagh, 2007), results within the field of behavioral health remain mixed. While several studies have found that clinician characteristics such as EBT attitudes, gender, and theoretical orientation influence the use of evidence-based treatments and implementation outcomes (Beidas, Edmunds, et al., 2014; Ditty, Landes, Doyle, \& Beidas, 2015), some studies have found that they do not affect implementation outcomes (Bearman et al., 2013; Lewis \& Simons, 2011). Therefore, additional research is necessary to understand the role of trainee characteristics on training outcomes.

Given the lack of research on consultation and the inconsistent findings related to individual trainee characteristics, the present study examined the consultation component of a multi-component training protocol for PCIT. The aims of the current study were twofold: (a) to examine if greater consultation call attendance predicts greater post-training clinician knowledge, skill, acceptability, feasibility, and (b) to investigate if clinician characteristics (caseload, licensure, baseline knowledge, years of experience, and experience with preschool children) influence these relationships.

\section{Method}

\section{Parent study}


The present study utilized data from a parent study funded by the National Institute of Mental Health (NIMH; R01 MH095750; A Statewide Trial to Compare Three Training Models for Implementing an EBT; PI: Herschell). The parent study is a statewide trial examining three implementation methods (training models) for PCIT including: (a) cascading training model (CM), a hierarchical training method, (b) distance-education (DE), an online-computer based course, and (c) learning collaborative (LC), which addresses the clinical, supervisor, administrator, and senior leader levels of an agency and emphasizes collaboration across agencies. The CM involves training senior clinicians within an organization, who then have the ability to train other clinicians within their organization. DE involves an individual clinician completing and interacting with a web-based training course. The LC model, originally developed by the Institute for Health Improvement, and adapted to behavioral health by the National Child Traumatic Stress Network, involves training multiple levels within an organization (e.g., clinicians, supervisors, senior leaders) with a specific focus on implementation within an organization. Fifty eligible agencies were randomized to one of the three aforementioned training conditions. The parent study examines clinician-level (e.g., knowledge), clinic-level (e.g., adoption), and client-level (e.g., disruptive behavior problems) outcomes. See Herschell and colleagues (2015) for additional details on the parent study.

\section{Participants}

Participants from the cascading condition of the parent study were included in the present study. To be included in the current study, clinicians had to (a) be currently employed at a clinic meeting inclusion criteria (see Herschell et al., 2015), (b) hold a master's- or doctoral-level degree, (c) be licensed in his/her field or receiving supervision from a licensed individual, (d) be able to provide treatment to families appropriate for PCIT, (e) have no previous training in PCIT 
but were receptive to training, and (f) be willing to participate in various study tasks (e.g., completing assessments, video-taping sessions).

Participants were 32 clinicians from 16 agencies, with two clinicians per agency, across the Commonwealth of Pennsylvania. Table 1 contains demographic information for the participants. Twenty nine (91\%) of the participants were female, 31 (97\%) of the participants were Caucasian, 32 (100\%) held a master's degree, and $11(34 \%)$ were professionally licensed.

\section{Procedure}

All procedures were approved by the University of Pittsburgh Medical Center's Institutional Review Board and West Virginia University’s Institutional Review Board. Clinicians completed questionnaires at four separate time-points: baseline, 6- (mid), 12-(post), and 24-months (1 year follow-up). Relevant to the proposed study, at baseline, clinicians completed a Clinician Background and Contact Information form. The Usage Rating ProfileIntervention scale was administered at 6-, and 12-months. The PCIT Coaches Quiz was completed across all time-points. The Clinician PCIT Skills Checklist was completed throughout training, consultation calls, and video-review of tapes that clinicians submitted.

Incentives were included for participation of both clinicians and agencies. Clinicians received free training, Continued Education Credits, and payment for completion of questionnaires ( $\$ 25$ for baseline and 6-month follow-up, $\$ 30$ for 12-month follow-up, and $\$ 40$ for 24-month follow-up). Agencies received free PCIT training for their clinicians and a stipend $(\$ 1,000)$ for initial startup costs and necessary equipment for utilizing PCIT in their organization.

Trainers. Three trainers who participated in the cascading training condition as part of a postdoctoral research training experience were included in the current study. Three trainers participated in the cascading condition and were balanced across training conditions and across 
consultation call groups (see Table 2). For the purposes of this study, the trainers and consultants were the same experts, and are hereby referred to as trainers. The trainers were Caucasian females in their late twenties and had extensive training and expertise in PCIT, disruptive behavior disorders, and child welfare. Two of the trainers received their Doctorate in Clinical Child Psychology, and one of the trainers received her Doctorate in School Psychology. All of the trainers were licensed or licensed eligible at the time of the training and consultation calls.

Training. The CM utilized in this study is consistent with PCIT International Training guidelines, and was carefully developed and operationalized through an expert consensus process with PCIT Master Trainers (Scudder \& Herschell, 2015). Training was conducted in four waves across the Commonwealth of Pennsylvania as training across the state could not be completed at once. See Figure 1 for a depiction of the wave approach to training counties in Pennsylvania. Training consisted of an initial 40 hours of face-to-face contact with two of three expert PCIT trainers, an advanced live training of 16 hours utilizing real cases six months after the initial training, and ongoing bi-weekly contact with a PCIT trainer over 12 months. A training integrity checklist was completed at each in-person training to assess that training content was administered according to protocol. Two clinicians from each organization were included in each training group, with each training group including an average of eight clinicians total $(S D=2.0)$.

Consultation Calls. Included in the 12-months of intensive training, clinicians had the opportunity to attend up to 24 1-hour consultation calls conducted by one of three expert consultants, who were also trainers for the in-person training. In total, 96 consultation calls were conducted across four consultation call groups. Trainers led consultation calls according to the PCIT Case Consultation Outline and Record, which aligns with Nadeem and colleagues' (2013) core components of consultation. 
Treatment. Parent-Child Interaction Therapy (PCIT; Eyberg \& Funderburk, 2011; McNeil \& Hembree-Kigin, 2010) is a "well-established" manualized EBT for youth ages 2.5- to 7-years-old with externalizing behavior problems (McNeil \& Hembree-Kigin, 2010). PCIT focuses on improving the quality of parent-child interactions and increasing child compliance, while also reducing disruptive behaviors such as aggression and defiance. PCIT is divided into two phases of treatment, Child-Directed Interaction (CDI) and Parent-Directed Interaction (PDI). During the CDI phase, parents learn non-directive play skills that work to engage the child in play and improve the quality of the parent-child relationship. During the PDI phase, parents include skills such as commands and consequences (e.g., time-out) with the goal of reducing disruptive behaviors and increasing compliance (Eyberg \& Funderburk, 2011; McNeil \& Hembree-Kigin, 2010).

\section{Measures}

Clinician Background Form. Basic demographic information for clinicians was collected utilizing the Clinician Background Form, adapted from an existing community practitioner demographic form (Kolko et al., 2012). This form consists of demographic items such as gender, level of education, licensure status, length of time employed at current organization, and geographic area of organization (e.g., rural). This form also contains items related to previous clinical experiences such as experience with behavioral parent training, recent trainings attended, and therapy experience with preschool children.

PCIT Case Consultation Outline \& Record. Trainers completed this measure following each consultation call to track clinician attendance and consultation content. This outline contains sections for trainers to indicate: (a) clinician attendance and participation, (b) clinician caseload, (c) trainer perspectives of the most impactful content areas on the consultation 
call, (d) clinician-reported barriers to implementation, (e) and amount of time engaged in different consultation content areas. Consultation content areas in the outline align with Nadeem and colleagues' (2013) examination of core components of the consultation process. Trainers estimate the amount of time spent engaging in various consultation activities including continued training on key topics, problem-solving implementation barriers (e.g., lack of referrals), reviewing cases, holding clinicians accountable (e.g., submitting parent-child assessments), building skills (e.g., role plays, feedback on skill demonstration), planning for sustainability, structuring the call (e.g., attendance), discussing administrative concerns (e.g., technical issues with website), and other topics.

PCIT Coaches Quiz. The PCIT Coaches Quiz (PCQ; Funderburk \& Nelson, 2014) measures clinician knowledge of PCIT treatment protocol and skills. This 24-item multiplechoice questionnaire includes items related to PCIT protocol, example scenarios, and evaluating mock in-session coding sheets. Scoring of this measure corresponds with the number of items that a clinician answers correctly, with scores ranging from 0 to 24 .

Clinician PCIT Skills Checklist. This 19-item checklist assesses skill and was developed according to competencies that align with the PCIT International Training Guidelines (2013). These competencies were reviewed and approved by an expert PCIT panel, and include a combination of evaluation at the in-person training, consultation calls, and video review. Completed by the PCIT trainer, this checklist addresses core competencies of PCIT including PCIT assessment, CDI skills, PDI skills, PCIT general skills, and completion of training cases. Trainers indicate the date by which each clinician achieved mastery on each core competency.

Usage Rating Profile-Intervention. The Usage Rating Profile-Intervention measure (URP-I; Chafouleas, Riley-Tillman, Briesch, \& Chanese, 2008) evaluates implementation 
outcomes. The URP-I is a 35-item 6-point Likert-type self-report measure, which ranges from 1 to 6 ("strongly disagree" to "strongly agree"), developed to understand intervention usage as determined by four subscales: acceptability, understanding, feasibility, and systems support. The acceptability and feasibility subscales were utilized as they are consistent with Proctor and colleagues' (2009) taxonomy of implementation outcomes. Results of an exploratory factor analysis support the measure's use of four subscales, and evidence for internal consistency reliability was established with an alpha greater than .70 for all sub-scales (Chafouleas, Briesch, \& Riley-Tillman, 2009).

\section{Data Analysis}

All data analyses were conducted in Statistical Package for the Social Sciences (version 24). For Aim 1, descriptive statistics were first calculated to understand overall consultation content over the course of the 24 calls. To determine if consultation content changed over time, the 24 consultation calls were divided into quarters (e.g., six calls per quarter), and the same descriptive statistics were calculated for each quarter of consultation calls. By dividing the consultation content into quarters, visual comparisons of means between quarters (e.g., first six calls compared to final six calls) were made to determine if amount of time devoted to a content area (e.g., reviewing cases) increased over time.

To examine Aims 2 and 3 (whether attending more consultation calls is associated with greater knowledge change and post-training skill, acceptability, and feasibility), a series of linear regression analyses were conducted. First, four simple linear regression analyses were conducted to identify the impact of consultation call attendance alone in predicting knowledge change and post-training skill, acceptability, and feasibility. Second, four multiple linear regression analyses were conducted to determine if consultation call attendance with the inclusion of the clinician 
characteristics (e.g., caseload, licensure, baseline knowledge, years of experience, experience with preschool children) entered into the regression model uniquely predicted knowledge change, post-training skill, acceptability, and feasibility. Given that a change score was calculated for knowledge, baseline knowledge was not included in the multiple regression model predicting change in knowledge. The regression analyses were rerun predicting post-training knowledge while controlling for baseline knowledge and the results did not change. While latent growth curve models were also proposed to examine the influence on consultation call attendance on knowledge change, there was not enough power to conduct these analyses due to the small sample size. Polynomial regression analyses were also proposed, however, it was found that a linear model best fit the data.

Aim 4 analyzed if clinician characteristics moderated or mediated the association between consultation call attendance and knowledge change, post-training skill, acceptability, and feasibility. Conceptually, the following variables were hypothesized to be moderators that could impact the strength of consultation's effect on post-training outcomes: PCIT caseload, licensure, baseline knowledge, years of experience, and experience with pre-school children. See Figure 2 for an example of PCIT caseload as a moderator between consultation call attendance and skill. Moderation analyses were conducted using the PROCESS version 2.15 macro add-on developed by Preacher and Hayes (2004). In PROCESS, a series of hierarchical regression analyses are conducted with centered variables, and an interaction term is computed to determine moderation. For significant moderators, a Johnson-Neyman statistic was also utilized to assess the value of the moderator for which the relationship between the predictor and outcome variable became significant. 
Caseload was also hypothesized to be a mediator, where consultation call attendance influences post-training outcomes of knowledge, skill, acceptability, and feasibility through clinician caseload. Possible evidence for mediation was first examined through four linear regression analyses where consultation call attendance and caseload were entered as predictors into the model, and knowledge, skill, acceptability, and feasibility were utilized as the outcomes. If the unstandardized beta coefficient for consultation calls decreased with the inclusion of caseload in the model, suggesting potential mediation, a formal test of mediation would be conducted utilizing PROCESS. A mediation analysis utilizing the PROCESS macro add-on developed by Preacher and Hayes (2004) was calculated. The bootstrap method of testing mediation was utilized as opposed to Barron and Kenny's (1976) approach, given the conservative nature of Barron and Kenny's method, and the ability of PROCESS to provide a specific statistical test on the indirect effect of the mediator. As indicated by Preacher and Hayes (2004), evidence for mediation is found when the confidence interval (CI) for the indirect effect does not contain zero. In the present analyses, 1000 bootstrap samples were requested to estimate the $95 \%$ CI to estimate significant direct and indirect effects.

\section{Results}

\section{Descriptive Statistics}

Preliminary analyses were conducted to determine if violations occurred in normality, linearity, homoscedasticity, and multicollinearity. Skewness and kurtosis values fell within acceptable limits between -2 and +2 indicating normality of data (George \& Mallery, 2010). Scatterplots indicated a linear relationship between predictor variables and the outcomes of knowledge, skill, acceptability and feasibility. Homoscedasticity was examined through visual inspection of residual scatterplots with standardized predicted values plotted against standardized 
residual values. An analysis of variance inflation factor and tolerance levels for the regression models indicated no violations in multicollinearity.

Means and correlations between all study variables are depicted in Table 3. Participants attended an average of $17.60(M=74 \%, S D=28.2 \%)$ of 24 consultation calls. Participants had an average PCIT caseload of 3.81 families $(S D=4.68)$, with five $(15.6 \%)$ clinicians completing two cases over the course of training and consultation calls. Mean knowledge percentage scores increased from baseline $(M=39.7, S D=10.40)$ to post-training $(M=72.1, S D=13.60)$.

Participants met an average of $10.25(S D=3.65)$ competencies throughout training. The average acceptability rating for participants was $69.4(S D=6.40)$, while the average feasibility rating for participants was $39.74(S D=10.43)$.

\section{Consultation Call Content}

On average, $5.10(S D=1.20)$ content topics were discussed per consultation call. Figure 3 depicts the average amount of time spent devoted to each content area. Consultants spent an average of 19.32 minutes $(S D=7.54)$ reviewing cases, 12.65 minutes $(S D=7.00)$ on continued training topics, 12.13 minutes $(S D=3.39)$ structuring the call, 5.85 minutes $(S D=2.26)$ problem-solving implementation barriers, 3.23 minutes $(S D=2.00)$ building skills, 1.47 minutes $(S D=.79)$ on other topics, and less than one minute on administrative concerns $(M=.81, S D=$ $.52)$, sustainability planning $(M=.47, S D=.36)$, and holding clinicians accountable $(M=.18$, $S D=.18)$. Appendix A contains the results of Aim 1, analyzing how consultation call content changes over time.

\section{Consultation Call Attendance and Outcomes}

Consultation call attendance. Table 4 presents the results of the simple linear regression analyses. Consultation call attendance significantly predicted change in knowledge $(F(1,27)=$ 
$\left.9.90, \beta=.52, p<.01, R^{2}=.27\right)$, skill $\left(F(1,30)=23.10, \beta=.66, p<.001, R^{2}=.44\right)$, and acceptability $\left(F(1,28)=4.30, \beta=.36, p<.05, R^{2}=.13\right)$. Consultation call attendance did not significantly predict feasibility $(F(1,28)=.10, n s)$.

Consultation call attendance and caseload. In the multiple linear regression model (see Table 5), consultation call attendance and caseload together significantly predicted change in knowledge $\left(F(2,28)=5.55, p=.01, R^{2}=.30\right)$, however, only consultation call attendance uniquely predicted change in knowledge $(\beta=.44, p<.05)$. A regression model (see Table 6 ) including consultation call attendance and caseload also significantly predicted skill $(F(2,31)=$ $\left.27.5, p<.001, R^{2}=.65\right)$, with both consultation call attendance $(\beta=.45, p=.001)$ and caseload $(\beta=.51, p<.001)$ uniquely predicting skill. The multiple regression model predicting acceptability from consultation call attendance and caseload was not significant in predicting acceptability $(F(2,29)=2.59, n s)$ nor feasibility $(F(2,29)=.13, n s)$.

Consultation call attendance and clinician characteristics. A multiple linear regression model (see Table 7) predicting change in knowledge from consultation call attendance, caseload, years of experience, licensure, and therapy experience with preschool children was not significant $(F(5,28)=2.5, n s)$. The same multiple regression model predicting skill was significant $\left(F(6,30)=9.0, p<.001, R^{2}=.69\right)$, with consultation call attendance $(\beta=$ $.47, p<.01)$, and caseload uniquely predicting skill $(\beta=.54, p<.001)$. The multiple linear regression model was not significant in predicting acceptability $(F(6,28)=2.5, n s)$, or feasibility $(F(6,28)=.26, n s)$

\section{Moderation Analyses}

PCIT caseload was the only moderator found to be significant in the relationship between consultation call attendance and skill (see Figure 4). At step 1 of the hierarchical linear 
regression, consultation call attendance and caseload significantly predicted skill $(F(2,32)=$ $22.5, p<.001, R^{2}=.71$, with consultation call attendance uniquely predicting skill $(\beta=.45, p<$ $.01)$. At step 2, the interaction term (consultation call attendance * caseload) was entered into the model, and significantly predicted skill $(b=.02, p<.05)$. The Johnson-Neyman statistic was not significant, indicating that there was not a significant transition level of the moderator for which the interaction became significant.

\section{Mediation Analyses}

Evidence for potential mediation was suggested by a decrease in the unstandardized beta coefficient between two regression models predicting skill. In the first regression model, consultation call attendance significantly predicted skill $(F(1,30)=23.1, b=.09, p<.001)$. $F(1,30)=23.1, \beta=.66, p<.001, R^{2}=.44$. In the second model, consultation call attendance and caseload significantly predicted skill $\left(F(2,31)=27.5, p<.001, R^{2}=.65\right)$. In this model, consultation call attendance uniquely predicted skill $(b=.06, p<.01)$, demonstrating a decrease in the unstandardized beta coefficient from the first regression.

A formal test of mediation was conducted in PROCESS through a series of regression analyses. The results of the mediation analysis are displayed in Figure 5. Consultation call attendance significantly predicted caseload $\left(F(1,30)=6.2, b=.07, p<.05, R^{2}=.17\right)$. Consultation call attendance and caseload significantly predicted skill $(F(2,31)=27.5, p<.001$, $\left.R^{2}=.65\right)$. An analysis of significant direct effects indicated that consultation call attendance had a significant direct effect on skill, $b=.06, p<.001$. The mediation test indicated a significant indirect effect of caseload on skill, $b=.06,95 \%$ CI $[.01, .05]$.

\section{Discussion}

\section{Overall Findings}


Although brief workshops and trainings are commonly utilized training methods, the current study lends support to a growing body of literature demonstrating the importance of ongoing support as a component of training protocols for EBTs (Herschell et al., 2010; Lyon, Stirman, Kerns, \& Bruns, 2011). Our initial hypotheses predicted that greater consultation call attendance would be associated with greater change in knowledge and post-training skill, acceptability, and feasibility. These hypotheses were partially supported. While simple linear regression analyses supported the importance of consultation call attendance in predicting knowledge, skill, and acceptability, when additional clinician variables were included in the model, consultation call attendance only significantly predicted post-training skill. Overall, these findings suggest that consultation call attendance is uniquely associated with increased clinician skill in PCIT, despite various clinician characteristics.

However, the relation between consultation call attendance and skill was qualified by a significant interaction between consultation call attendance and caseload. This interaction suggests that clinicians with a high caseload who attend a greater number of consultation calls reap the most benefits in skill. Further, this implies that increases in clinician skill occur as a result of the interplay between training and experience with cases. Additionally, clinicians with a low caseload demonstrated a similar skill level regardless of the amount of consultation calls attended, lending support to this finding. Thus, increasing clinician skill requires a combination of ongoing support as well as the opportunity to utilize newly learned skills with clients. However, clinicians who have a high caseload, but attend few consultation calls demonstrated the lowest skill level in the current study. One possible explanation for this finding is that clinicians who had a high PCIT caseload but did not attend additional consultation calls may be 
overwhelmed with other areas of their caseload that were not measured as part of the study, and therefore, could not attain adequate skills related to PCIT.

Interestingly, this study did not find support for the importance of consultation calls in predicting the implementation outcomes of feasibility and acceptability. It was hypothesized that clinicians who attended a greater number of consultation calls would perceive PCIT as being more feasible and acceptable to utilize with their clients. However, this study did not find evidence in support of this hypothesis. It is possible that improving implementation outcomes may be more related to organizational characteristics as opposed to training design factors. Research is beginning to reveal that organizational characteristics, such as organizational climate and supervisory support, influence the degree to which a clinician implements a treatment (Beidas et al., 2014; Ditty et al., 2015; Glisson, 2002). These organizational-level characteristics may be more important in influencing implementation outcomes than clinician-level characteristics or training design factors. Therefore, additional research should explore if organizational factors are also related to the implementation outcomes of feasibility and acceptability.

While the present study did find PCIT caseload to be a moderator of consultation call attendance and skill, no other significant moderators were found. This finding differs from previous research which suggests the importance of clinician characteristics in the implementation of evidence-based treatments (Beidas et al., 2014; Lewis \& Simons, 2011; Velada et al., 2007). Prior research has not examined the influence of clinician characteristics on consultation call outcomes. However, the growing research dedicated to individual clinician characteristics suggests that it should be an area for future research within the training literature.

\section{Limitations}


Directionality of associations. This study has examined the correlational association between consultation call attendance, clinician variables, and clinician and implementation outcomes. Although this study presents evidence in support of the importance of consultation call attendance and caseload on clinician outcomes, the nature of this association is unclear. The current study hypothesized that consultation call attendance is a predictor of clinician caseload, suggesting that clinicians who attend more consultation calls have a higher caseload. However, it is also possible that clinician caseload predicts consultation call attendance. In this example, clinicians who are treating more families are likely to attend more calls, given that a core component of consultation calls involves reviewing cases. Similarly, clinicians who have a small number of cases are more likely to attend fewer consultation calls. Additional research should seek to clarify the directionality of this association.

Measurement. Several measures included in the current study (e.g., Clinician PCIT Skills Checklist, PCIT Case Consultation Record) have not demonstrated adequate psychometric evidence, limiting the interpretations that can be drawn. While the Clinician PCIT Skills Checklist was utilized given that it aligns with PCIT International's Training Guidelines (2009) and certification process, it has not demonstrated adequate reliability or validity in assessing clinician skill. The field of implementation science as a whole has been limited by a lack of measures that have appropriate psychometric properties, in addition to relying upon studydeveloped measures (Martinez, Lewis, \& Weiner, 2014). The lack of adequate measurement weakens the findings of the current study.

Additional problems with the Clinician PCIT Skills Checklist may have further hindered results of the study. First, two items included in this measure were identified as being met during consultation calls by the trainers. These items include attending $80 \%$ of consultation calls and 
completing two PCIT cases. The first item is problematic given that consultation call attendance is utilized as both a predictor and as an item of the composite outcome measure, potentially inflating the impact of consultation call attendance on skill. The second item, completing two PCIT cases, also may have been completed without the trainer's knowledge. For example, it is possible that a clinician completed two PCIT cases but did not attend any consultation calls, and therefore the trainer was unaware of this completion.

In addition to the measurement of competencies on consultation calls, several items were completed through video review of sessions with clients (e.g., CDI Coach Session). However, if clinicians were unable to enroll clients on their caseload into the study due to a variety of reasons (e.g., lack of consent, lack of referrals), clinicians were unable to meet competencies requiring video review. In order to reduce this issue, during the consultation phase, trainers attempted to problem-solve the barrier of clinicians not receiving enough referrals. Additionally, clinicians were encouraged to enroll a large amount of families in the study in order to submit videos given the possibility of families dropping out of treatment or not consenting to the study.

Nesting. The current study involves clinicians, who are nested within a supervisor, who is nested within an organization. However, given the small sample size utilized in the current study, hierarchical linear modeling procedures could not be utilized. Therefore, the interpretations that can be made from this study are limited in that the variations in clinician skill may also be due in part to supervisor or organizational factors.

Sample size. While a power analysis was conducted and the sample size was determined to be appropriate for the simple linear regression analyses described previously, it is possible that the study was underpowered to detect moderation effects. Further research should examine how therapist characteristics influence the association between consultation call attendance and 
outcomes. Additionally, the current study was underpowered to perform growth curve analyses that may have been more amenable to our hypotheses in examining change over time as a result of consultation call attendance.

\section{Future Directions}

These findings demonstrate the importance of analyzing training design components that influence clinician knowledge and skill outcomes. The consultation calls in the current study primarily focused on reviewing clinician cases, however, additional research should focus on targeting which content areas optimize training outcomes. For example, some research has found support for the importance of skill-building activities in supervision for promoting clinician fidelity (Beidas, Cross, \& Dorsey, 2014). The current study found that consultants did not devote substantial time to building skills, however, it may be an important aspect of ongoing support. Additional research should focus on the mechanisms and content areas that underlie the effectiveness of ongoing support strategies such as consultation. Additionally, the current study examined one form of ongoing support, consultation, in regards to these outcomes. While consultation is widely utilized in large-scale training initiatives, other forms of ongoing support (e.g., supervision) should also be examined.

In addition, the present study has highlighted the importance of the training design input factor in producing positive training outcomes. Although research has examined how individual clinician characteristics (Beidas, Edmunds, et al., 2014; Lewis \& Simons, 2011; Velada et al., 2007) and organizational characteristics (Beidas et al., 2015; Ditty et al., 2015) influence implementation outcomes, these input factors remain largely unstudied in relation to training clinicians in EBTs. Further research should examine other training input factors of Baldwin and Ford's (1988) model that influence training outcomes for clinicians. 
Prior research on training outcomes has generally isolated one of Baldwin and Ford's (1988) training input factors when examining outcomes. This study attempted to understand the interaction between training design and individual trainee characteristics by examining moderators of consultation's effectiveness. While PCIT caseload was found to be a moderator of consultation's effectiveness, it is likely that other individual trainee characteristics or organizational factors may moderate this relationship. Additional research should seek to disentangle the complex interactions between training design, individual trainee characteristics, and organizational characteristics that influence training outcomes. Understanding the interface between training design and real-world implementation settings may result in improved training effectiveness, ultimately leading to the increased availability and accessibility of EBTs in community settings. 


\section{References}

Baldwin, T. T., \& Ford, J. K. (1988). Baldwin \& Ford 1988 training transfer model. Personnel Psychology, 41(1), 63-104.

Bearman, S. K., Weisz, J. R., Chorpita, B. F., Hoagwood, K., Ward, A., Ugueto, A. M., \& Bernstein, A. (2013). More practice, less preach? The role of supervision processes and therapist characteristics in EBP implementation. Administration and Policy in Mental Health and Mental Health Services Research, 40(6), 518-529. http://doi.org/10.1007/s10488-013-0485-5

Beidas, R. S., Cross, W., \& Dorsey, S. (2014). Show Me, Don’t Tell Me: Behavioral Rehearsal as a Training and Analogue Fidelity Tool. Cognitive and Behavioral Practice, 21(1), 1-11. http://doi.org/10.1016/j.pestbp.2011.02.012.Investigations

Beidas, R. S., Edmunds, J., Ditty, M., Watkins, J., Walsh, L., Marcus, S., \& Kendall, P. (2014). Are Inner Context Factors Related to Implementation Outcomes in Cognitive-Behavioral Therapy for Youth Anxiety? Administration and Policy in Mental Health and Mental Health Services Research. http://doi.org/10.1007/s10488-013-0529-x

Beidas, R. S., Edmunds, J. M., Marcus, S. C., \& Kendall, P. C. (2012). Training and consultation to promote implementation of an empirically supported treatment: a randomized trial. Psychiatric Services (Washington, D.C.), 63(7), 660-5. http://doi.org/10.1176/appi.ps.201100401

Beidas, R. S., Marcus, S., Aarons, Gregory A., Hoagwood, Kimberly, Schoenwald, Sonja K., Evans, Arthur C., ... Mandell, D. S. (2015). Individual and organizational factors related to community clinicians' use of therapy techniques in a large public mental health system. JAMA Pediatrics, 34(3), 355-368. 
http://doi.org/10.3109/10641955.2015.1046604.Association

Caplan, G. (1970). The theory and practice of mental health consultation. New York, NY: Basic Books.

Chafouleas, S. M., Riley-Tillman, T. C., Briesch, A. M., \& Chanese, J. M. (2008). Generating usable knowledge: Initial development and validation of the Usage Rating Profile for Interventions (URP-I). Canadian Journal of School Psychology, 23(2), 175-189. doi:10.1177/0829573508323989

Ditty, M. S., Landes, S. J., Doyle, A., \& Beidas, R. S. (2015). It Takes a Village: A Mixed Method Analysis of Inner Setting Variables and Dialectical Behavior Therapy Implementation. Administration and Policy in Mental Health and Mental Health Services Research. http://doi.org/10.1007/s10488-014-0602-0

Edmunds, J. M., Beidas, R. S., \& Kendall, P. C. (2013). Dissemination and implementation of evidence-based practices: Training and consultation as implementation strategies. Clinical Psychology: Science and Practice, 20(2), 152-165. http://doi.org/10.1111/cpsp.12031

Eyberg, S. \& Funderburk, B. (2011). Parent-child interaction therapy protocol. Gainesville, FL: PCIT International Publishing.

Facts on children's mental health in America. (2010) Retrieved from http://www2.nami.org/Template.cfm?Section=federal_and_state_policy_legislation\&tem plate $=/$ ContentManagement/ContentDisplay.cfm\&ContentID $=43804$.

Funderburk B, Nelson M. PCIT Coaches Quiz. 2014.

Glisson, C. (2002). The Organizational Context of Children's Mental Health Services. Clinical Child and Family Psychology Review, 5(4).

Glisson, C., \& Schoenwald, S. K. (2005). The ARC organizational and community intervention 
strategy for implementing evidence-based children's mental health treatments. Mental Health Services Research, 7(4), 243-259. http://doi.org/10.1007/s11020-005-7456-1

Herschell, A. D., Kolko, D. J., Baumann, B. L., \& Davis, A. C. (2010). The role of therapist training in the implementation of psychosocial treatments: A review and critique with recommendations. Clinical Psychology Review, 30(4), 448-466.

http://doi.org/10.1016/j.cpr.2010.02.005

Herschell, A. D., Kolko, D. J., Scudder, A. T., Taber-Thomas, S., Schaffner, K. F., Hiegel, S. A., ... Mrozowski, S. (2015). Protocol for a statewide randomized controlled trial to compare three training models for implementing an evidence-based treatment. Implementation Science, 10(1), 133. http://doi.org/10.1186/s13012-015-0324-z

Jensen-Doss, A., Hawley, K. M., Lopez, M., \& Osterberg, L. D. (2009). Using evidence-based treaments: The experiences of youth providers working under a mandate. Professional Psychology: Research and Practice, 40(4), 417-424.

Kataoka, S. H., Zhang, L., \& Wells, K. B. (2002). Unmet need for mental health care among U.S. children: Variation by ethnicity and insurance status. American Journal of Psychiatry, 159(9), 1548-1555. http://doi.org/10.1176/appi.ajp.159.9.1548

Kolko, D. J., Baumann, B. L., Herschell, A. D., Hart, J. A., Holden, E. A., \& Wisniewski, S. R. (2012). Implementation of AF-CBT by community practitioners serving child welfare and mental health: A randomized trial. Child Maltreatment, 1077559511427346.

Lewis, C. C., \& Simons, A. D. (2011). A Pilot Study Disseminating Cognitive Behavioral Therapy for Depression: Therapist Factors and Perceptions of Barriers to Implementation. Administration and Policy in Mental Health and Mental Health Services Research, 38(4), 324-334. http://doi.org/10.1007/s10488-011-0348-x 
Lyon, A. R., Stirman, S. W., Kerns, S. E. U., \& Bruns, E. J. (2011). Developing the mental health workforce: Review and application of training approaches from multiple disciplines. Administration and Policy in Mental Health and Mental Health Services Research, 38(4), 238-253. http://doi.org/10.1007/s10488-010-0331-y

Martinez, R. G., Lewis, C. C., \& Weiner, B. J. (2014). Instrumentation issues in implementation science. Implementation Science, 9, 118. http://doi.org/10.1186/s13012-014-0118-8

McNeil, C. B., \& Hembree-Kigin, T. L. (2010). Parent-Child Interaction Therapy (2 ${ }^{\text {nd }}$ ed.), New York: Springer.

Nadeem, E., Gleacher, A., \& Beidas, R. S. (2013). Consultation as an implementation strategy for evidence-based practices across multiple contexts: Unpacking the black box. Administration and Policy in Mental Health and Mental Health Services Research, 40(6), $439-450$.

Preacher, K. J., \& Hayes, A. F. (2004). SPSS and SAS procedures for estimating indirect effects in simple mediation models. Behavior Research Methods, Instruments, and Computers, $36,717-731$.

Rieckman, T., Bergmann, L., \& Rasplica, C. (2013). Legislating Clinical Practice: Counselor Responses to an Evidence-based Practice Mandate. Journal of Psychoactive Drugs, 1(7), 27-39. http://doi.org/10.1016/j.cbpra.2012.03.004.Training

Rodríguez, A., Southam-Gerow, M. A., O’Connor, M. K., \& Allin, R. B. (2014). An Analysis of Stakeholder Views on Children's Mental Health Services. Journal of Clinical Child \& Adolescent Psychology, 43(6), 862-876. http://doi.org/10.1080/15374416.2013.873982

Schoenwald, S. K., Chapman, J. E., Sheidow, A. J., \& Carter, R. E. (2009). Long-term youth criminal outcomes in MST transport: the impact of therapist adherence and organizational 
climate and structure. Journal of Clinical Child and Adolescent Psychology, 38(1), 91-105. http://doi.org/10.1080/15374410802575388

Scudder, A. T., \& Herschell, A. D. (2015). Building an evidence-base for the training of evidence-based treatments in community settings: Use of an expert-informed approach. Children and Youth Services Review, 55, 84-92. http://doi.org/10.1016/j.childyouth.2015.05.003

Sholomskas, D. E., Syracuse-Siewert, G., Rounsaville, B. J., Ball, S. a, Nuro, K. F., \& Carroll, K. M. (2005). We don't train in vain: a dissemination trial of three strategies of training clinicians in cognitive-behavioral therapy. Journal of Consulting and Clinical Psychology, 73(1), 106-15. http://doi.org/10.1037/0022-006X.73.1.106

Silverman, W. K., Pina, A. A., \& Viswesvaran, C. (2008). Evidence-based psychosocial treatments for phobic and anxiety disorders in children and adolescents. Journal of Clinical Child and Adolescent Psychology, 37(1), 105-130. http://doi.org/10.1080/15374410701817907

Velada, R., Caetano, A., Michel, J. W. J. W., Lyons, B. D., \& Kavanagh, M. J. (2007). The effects of training design, individual characteristics and work environment on transfer of training. International Journal of Training and Development, 11(4), 282-294. http://doi.org/10.1111/j.1468-2419.2007.00286.x

Waldron C. W., H. B. . T. (2008). Evidence-based psychosocial treatments for adolescent substance abuse. Journal of Clinical Child and Adolescent Psychology, 37(1), 238-261. http://doi.org/10.1080/15374410701820133

Wampold, B. E., Budge, S. L., Laska, K. M., del Re, A. C., Baardseth, T. P., Fluckiger, C., ... Gunn, W. (2011). Evidence-based treatments for depression and anxiety versus treatment- 
as-usual: A meta-analysis of direct comparisons. Clinical Psychology Review, 31(8), 13041312. http://doi.org/10.1016/j.cpr.2011.07.012

Weissman, M. M., Verdeli, H., Gameroff, M. J., Bledsoe, S. E., Betts, K., Mufson, L., ... Wickramaratne, P. (2006). National survey of psychotherapy training in psychiatry, psychology, and social work. Archives of General Psychiatry, 63(8), 925-934. http://doi.org/10.1016/S0084-3970(08)70650-9

Wells, K. C., \& Egan, J. (1988). Social-Learning and Systems Family-Therapy for Childhood Oppositional Disorder - Comparative Treatment Outcome. Comprehensive Psychiatry, 29(2), 138-146. http://doi.org/10.1016/0010-440x(88)90006-5 
Table 1

Demographic Information $(n=32)$

\begin{tabular}{lcc}
\hline Variable & $\mathrm{N}$ & $\%$ \\
\hline Gender & & \\
$\quad$ Male & 3 & 9 \\
$\quad$ Female & 29 & 91 \\
Race & & \\
$\quad$ Caucasian & 31 & 97 \\
$\quad$ Unknown/Not Reported & 1 & 3 \\
Education & & \\
$\quad$ Masters degree & 32 & 100 \\
Professional licensed & & \\
$\quad$ Yes & 11 & 34 \\
$\quad$ No & 21 & 66 \\
\hline
\end{tabular}




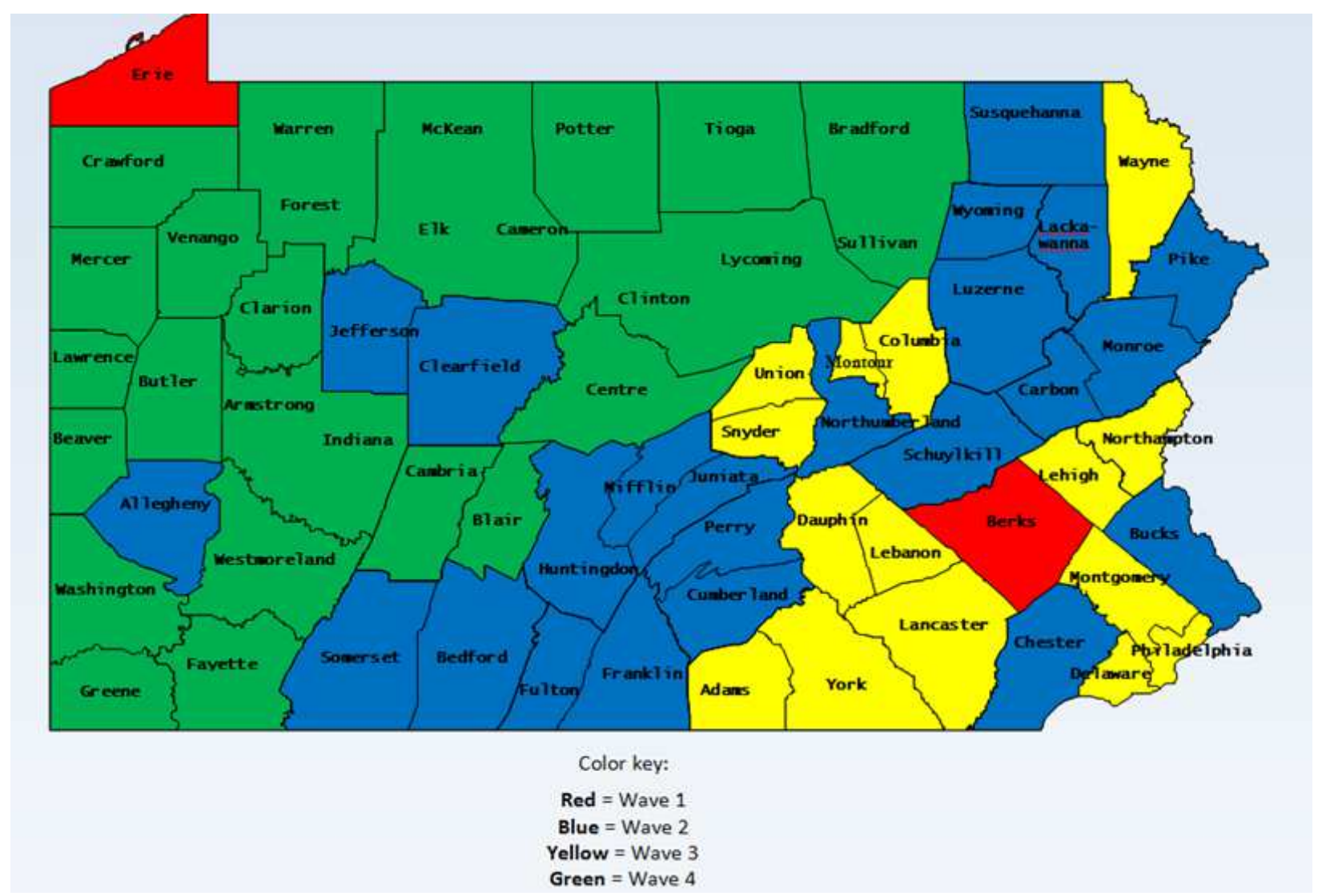

Figure 1. Training was conducted in four waves across the Commonwealth of Pennsylvania. 
Table 2

Trainer Balance across Waves of Training

\begin{tabular}{|c|c|c|c|c|c|c|c|c|}
\hline \multicolumn{3}{|c|}{ Wave 1} & \multicolumn{2}{|c|}{ Wave 2} & \multicolumn{2}{|c|}{ Wave 3} & \multicolumn{2}{|c|}{ Wave 4} \\
\hline Trainer & Training & Consultation & Training & Consultation & Training & Consultation & Training & Consultation \\
\hline 1 & $\mathrm{X}$ & $\mathrm{X}$ & & & $\mathrm{X}$ & & & \\
\hline 2 & & & & & $\mathrm{X}$ & $\mathrm{X}$ & $\mathrm{X}$ & \\
\hline 3 & $\mathrm{X}$ & & $\mathrm{X}$ & $X$ & & & $\mathrm{X}$ & $\mathrm{X}$ \\
\hline
\end{tabular}






Figure 2. The hypothesized moderating effect of PCIT caseload between consultation call attendance and skill 
Table 3

Bivariate Correlations between all Study Variables $(n=32)$

\begin{tabular}{|c|c|c|c|c|c|c|c|c|c|c|}
\hline Variables & $M(S D)$ & 1 & 2 & 3 & 4 & 5 & 6 & 7 & 8 & 9 \\
\hline 1. Consult. attendance & $74.06(28.16)$ & & & & & & & & & \\
\hline 2. Baseline knowledge & $39.74(10.43)$ & -.07 & & & & & & & & \\
\hline 3. Years exp. & $10.22(7.47)$ & .18 & -.10 & & & & & & & \\
\hline 4. Therapy exp. preschool children & $2.53(.98)$ & .08 & -.04 & .03 & & & & & & \\
\hline 5. Licensure & $.34(.48)$ & .03 & -.01 & $.40 *$ & .22 & & & & & \\
\hline 6. PCIT caseload & $3.81(4.68)$ & $.41 *$ & .14 & .17 & .02 & -.16 & & & & \\
\hline 7. Knowledge change & 32.97 (13.29) & $.52 * *$ & -.35 & $.38 *$ & .26 & .13 & $.37 *$ & & & \\
\hline 8. Skill & $10.25(3.65)$ & $.66^{*}$ & .08 & .21 & .03 & .10 & $.70 *$ & $.50 * *$ & & \\
\hline 9. Acceptability & $69.40(6.35)$ & $.36^{*}$ & -.01 & -.10 & .31 & -.27 & .31 & $.40 *$ & .24 & \\
\hline 10. Feasibility & $36.13(6.16)$ & -.06 & .22 & -.22 & -.06 & -.11 & .05 & -.22 & .04 & $.51 * *$ \\
\hline
\end{tabular}

$* p<.05, * * p<.01$ 




Figure 3. Average percentage of time devoted to consultation call topics across all consultation calls. 
Table 4

Simple Linear Regression Analyses Predicting Knowledge Change, Skill, Acceptability, and Feasibility from Consultation Call Attendance

\begin{tabular}{lccccc}
\hline & $B$ & $S E(B)$ & $\beta$ & $p$ & $R^{2}$ \\
\cline { 2 - 6 } Variable & & & & & \\
Knowledge change & .24 & .08 & .52 & $.00 * *$ & .269 \\
Skill & .09 & .02 & .66 & $.00 * * *$ & .435 \\
Acceptability & .08 & .04 & .36 & .048 & .132 \\
Feasibility & -.01 & .04 & -.06 & .75 & .004 \\
$*^{*} p<.05, * * p<.01, * * * p<.001$ & & & & &
\end{tabular}


Table 5

Multiple Linear Regression Analysis Predicting Knowledge Change from Consultation Call Attendance and PCIT Caseload

\begin{tabular}{lccccc}
\hline & $B$ & $S E(B)$ & $\beta$ & $p$ & $R^{2}$ \\
\cline { 2 - 6 } Model & & & & $.01^{*}$ & .299 \\
$\quad$ Consultation attendance & .20 & .08 & .44 & $.02^{*}$ & \\
PCIT caseload & .53 & .50 & .19 & .30 & \\
\hline$* p<05$ & & & &
\end{tabular}

${ }^{*} p<.05$ 
Table 6

Multiple Linear Regression Analysis Predicting Skill from Consultation Call Attendance and PCIT Caseload

\begin{tabular}{lccccc}
\hline & $B$ & $S E(B)$ & $\beta$ & $p$ & $R^{2}$ \\
\cline { 2 - 6 } Model & & & & $.00^{* * *}$ & .654 \\
$\quad$ Consultation attendance & .06 & .02 & .45 & $.00^{* *}$ & \\
PCIT caseload & .40 & .10 & .51 & $.00^{* * *}$ & \\
\hline$* * p<.01,{ }^{* * *} p<.001$ & & & & &
\end{tabular}


Table 7

Multiple Linear Regression Analyses Predicting Skill from Consultation Call Attendance and Clinician Variables

\begin{tabular}{lccccc}
\hline & $B$ & $\begin{array}{c}S E \\
(B)\end{array}$ & $\beta$ & $p$ & $R^{2}$ \\
\cline { 2 - 6 } Model & & & & & $.00 * * *$ \\
$\quad$ Baseline knowledge & & & & & .691 \\
Consultation attendance & .06 & .02 & .47 & $.00 * *$ & \\
Licensure & 1.36 & 1.02 & .17 & .20 & \\
PCIT caseload & .42 & .10 & .54 & $.00 * * *$ & \\
Therapy exp. preschool children & -.57 & .46 & -.15 & .23 & \\
Years experience & -.03 & .07 & -.05 & .67 & \\
\hline
\end{tabular}

$* p<.05, * * p<.01, * * * p<.001$ 


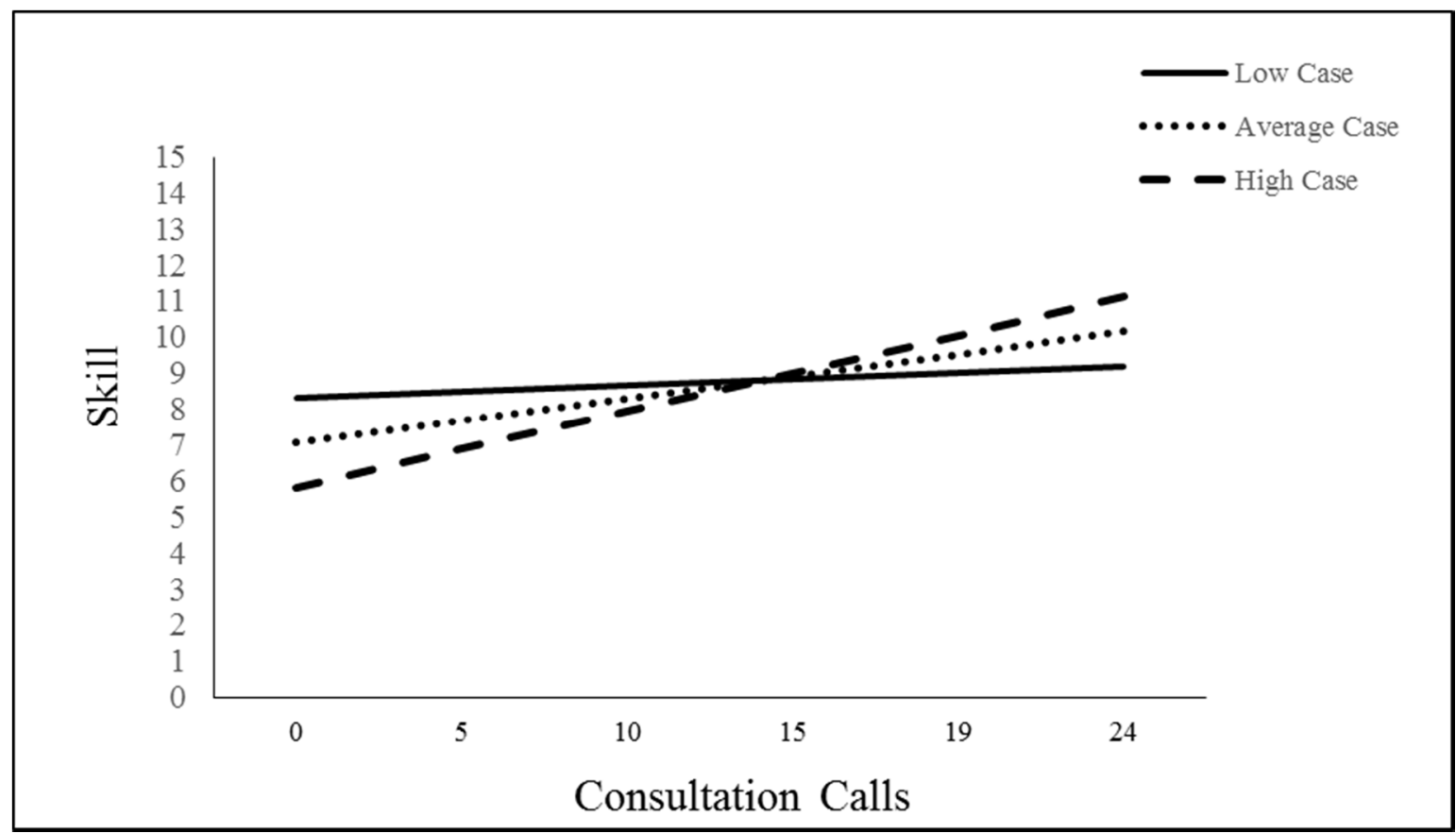

Figure 4. Results of the moderating effect of caseload on the relation between consultation call attendance and skill. 




Figure 5. The indirect effect of caseload on the relation between consultation call attendance and skill. 


\section{Appendix A}

\section{Changes in Consultation Content over Time}

Figure A1 displays the consultation content over the first six consultation calls. The first six calls spent an average amount of time discussing the following topics: 17.53 minutes $(S D=$ $10.07)$ reviewing cases, 13.60 minutes $(S D=4.34)$ structuring the call, 12.17 minutes $(S D=$ 11.00) on continued training, 9.27 minutes $(S D=4.20)$ problem-solving implementation barriers, 2.10 minutes $(S D=2.22)$ discussing administrative concerns, 1.40 minutes $(S D=2.31)$ building skills, 1.23 minutes $(S D=1.21)$ on other topics, and 0 minutes holding clinicians accountable of planning for sustainability.

Figure A2 displays the consultation content covered between calls 7-12. These calls spent an average amount of time discussing the following topics: 18.07 minutes $(S D=6.94)$ reviewing cases, 11.97 minutes $(S D=7.27)$ on continued training, 11.73 minutes $(S D=2.78)$ structuring the call, 6.93 minutes $(S D=4.20)$ problem-solving implementation barriers, 4.33 minutes $(S D=$ 3.23) building skills, 1.71 minutes $(\mathrm{SD}=1.02)$ planning for sustainability, and less than one minute holding clinicians accountable $(M=.56, S D=.96)$ and other topics $(M=.22, S D=1.9)$.

Figure A3 displays the consultation content covered between calls 13-18. These calls spent an average amount of time discussing the following topics: 22.03 minutes $(S D=11.20)$ reviewing cases, 14.33 minutes $(S D=7.34)$ on continued training, 12.23 minutes $(S D=3.95)$ structuring the call, 4.42 minutes $(S D=3.49)$ problem-solving implementation barriers, 3.63 minutes $(S D=3.35)$ building skills, 2.00 minutes $(\mathrm{SD}=.58)$ on other topics, less than one minute discussing administrative concerns $(M=.83, S D=1.18)$ and planning for sustainability $(M=.28, S D=.48)$, and 0 minutes holding clinicians accountable. 
Figure A4 displays the consultation content covered over the last calls (calls 18-24). These calls spent an average amount of time discussing the following topics: 22.03 minutes (SD $=11.20)$ reviewing cases, 15.33 minutes $(S D=7.34)$ on continued training, 12.23 minutes $(S D=$ 3.95) structuring the call, 4.42 minutes $(S D=3.49)$ problem-solving implementation barriers, 3.63 minutes $(S D=3.35)$ building skills, 2.00 minutes $(\mathrm{SD}=.58)$ on other topics, less than one minute discussing administrative concerns $(M=.83, S D=1.18)$ and planning for sustainability $(M=.28, S D=.48)$, and 0 minutes holding clinicians accountable. 


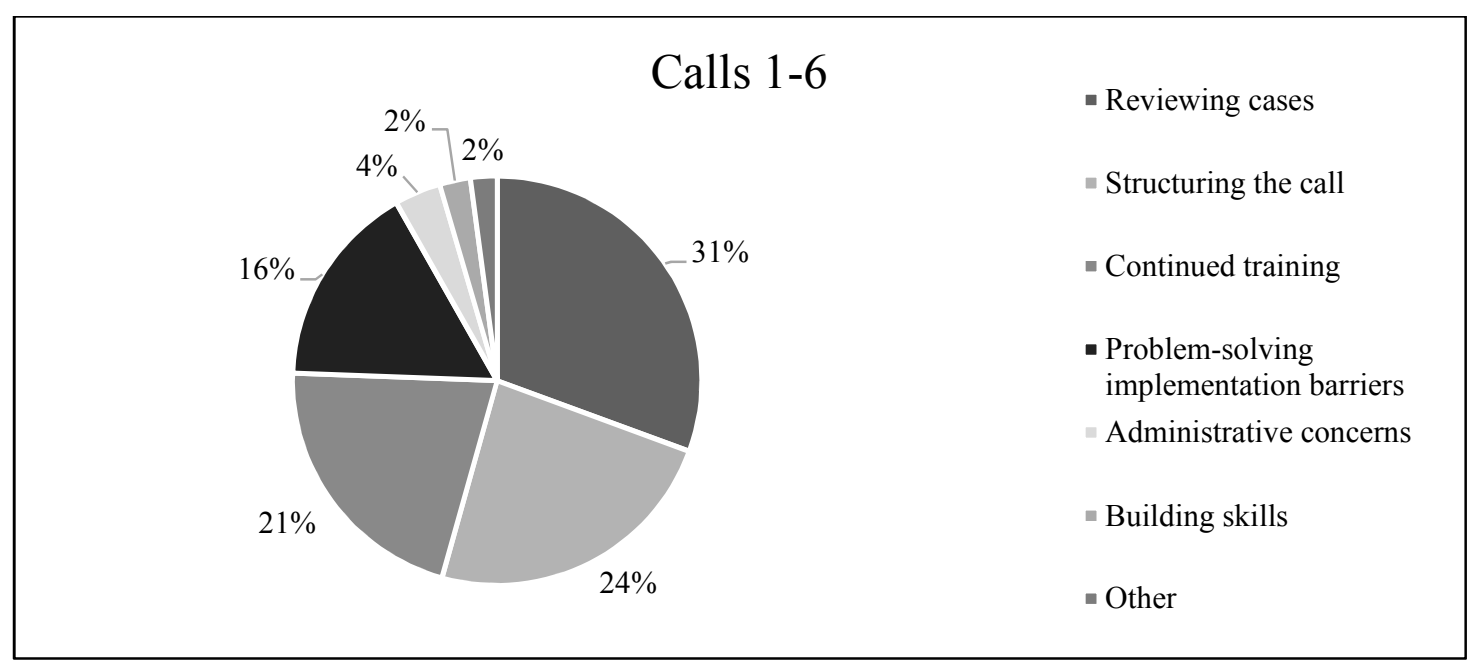

Figure A1. This pie chart depicts the average consultation call content covered within the first six calls. 




- Reviewing cases

- Continued training

- Structuring the call

- Problem-solving implementation barriers

Building skills

- Other

- Sustainability planning

- Holding clinicians accountable

Figure A2. This pie chart depicts the average consultation call content covered within the calls 7-

12. 


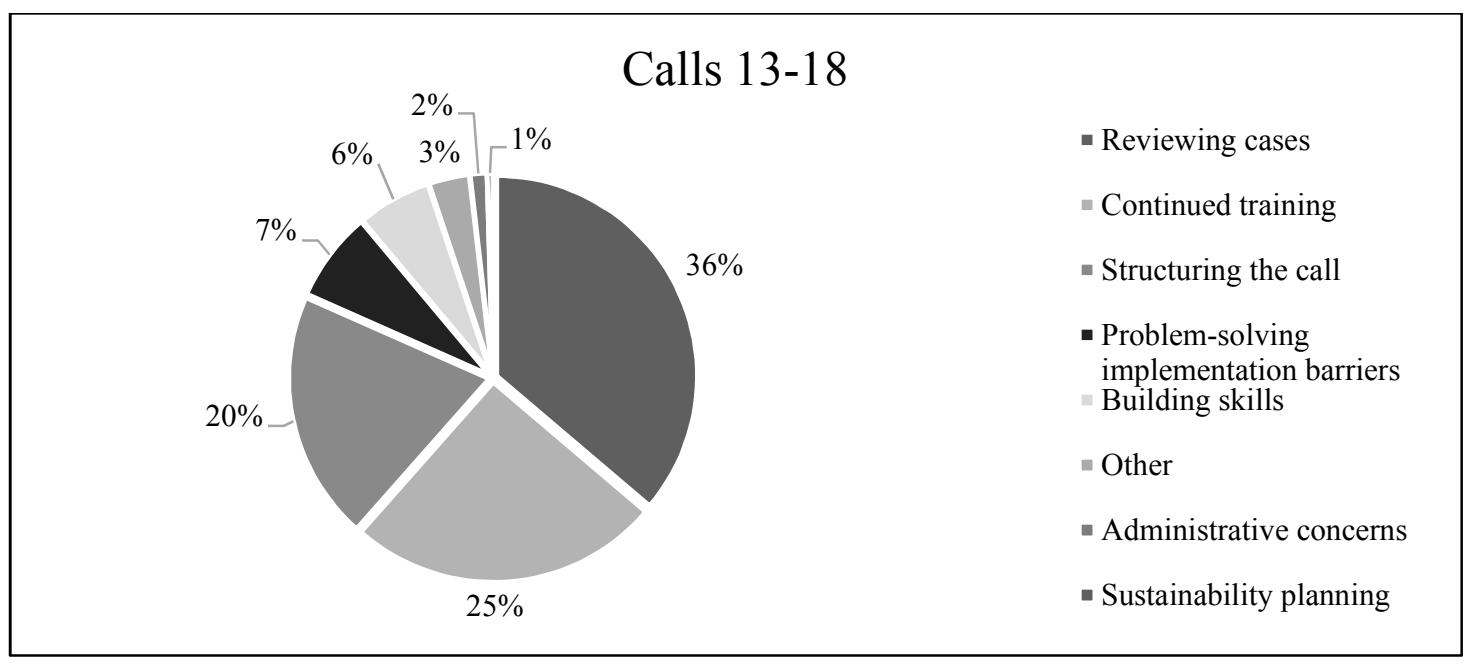

Figure A3. This pie chart depicts the average consultation call content covered within the calls $13-18$. 


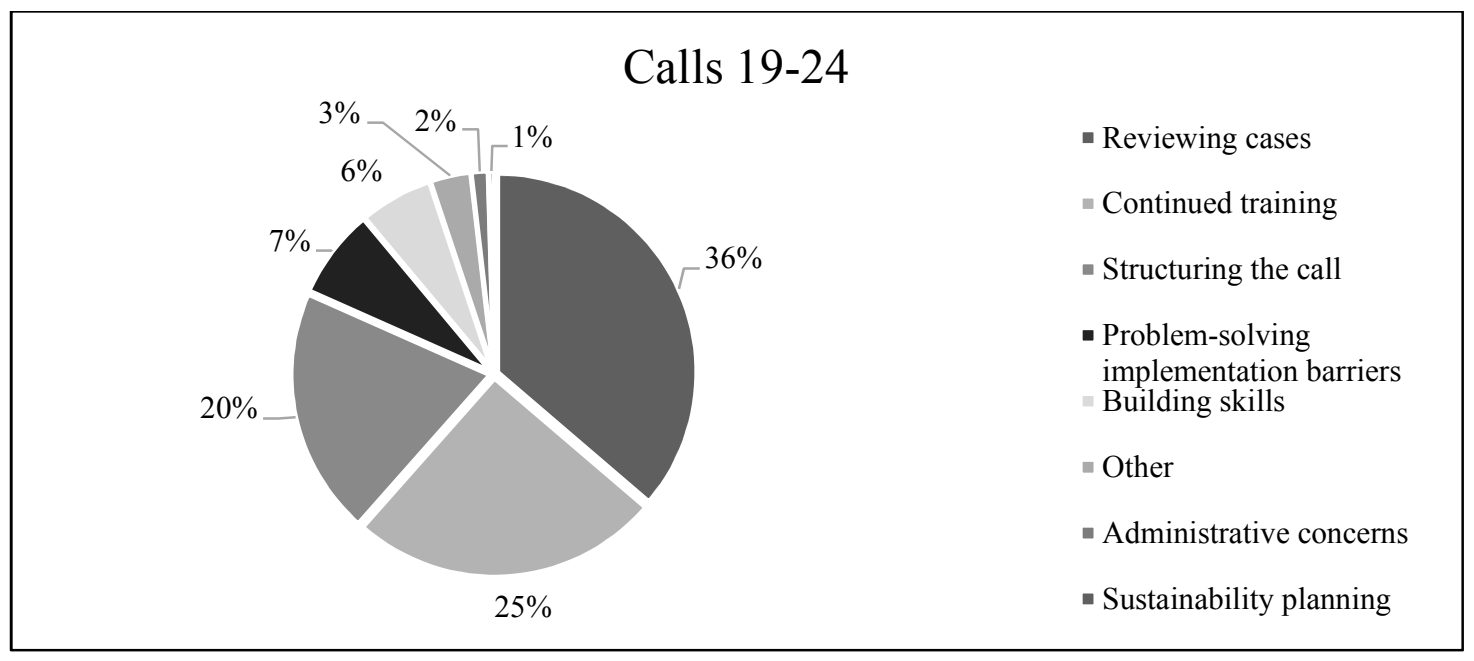

Figure A4. This pie chart depicts the average consultation call content covered within the calls $19-24$. 


\section{Appendix B}

\section{Linear Regression Analyses Utilizing Attending 80\% of Consultation Calls (PCIT International's Training Guidelines)}

\section{Attending 80\% of Consultation Calls, Caseload, and Clinician Characteristics Predicting Outcomes}

Consultation call attendance. Consultation call attendance significantly predicted change in knowledge $\left(F(1,28)=8.23, \beta=.48, p<.01, R^{2}=.233\right)$, skill $(F(1,31)=5.16, \beta=.38$, $\left.p<.05, R^{2}=.147\right)$, and acceptability $\left(F(1,28)=4.97, \beta=.39, p<.05, R^{2}=.148\right)$. Consultation call attendance did not significantly predict feasibility $(F(1,28)=.00, n s)$.

Consultation call attendance and caseload. In the multiple linear regression model, consultation call attendance and caseload together significantly predicted change in knowledge $\left(F(2,28)=4.85, p<.05, R^{2}=.272\right)$, however, only consultation call attendance uniquely predicted change in knowledge $(\beta=.40, p<.05)$. A regression model including consultation call attendance and caseload also significantly predicted skill $\left(\mathrm{F}(2,31)=23.30, p<.001, R^{2}=.616\right)$, with both consultation call attendance $(\beta=.39, \mathrm{p}<.01)$ and caseload $(\beta=.55, \mathrm{p}<.001)$ uniquely predicting skill. The multiple regression model predicting acceptability from consultation call attendance and caseload was not significant in predicting acceptability $(F(2,29)=2.89, n s)$ nor feasibility $(F(2,29)=.03, n s)$.

Consultation call attendance and clinician characteristics. A multiple linear regression model predicting change in knowledge from consultation call attendance, caseload, years of experience, licensure, and therapy experience with preschool children was not significant $(F(5,28)=2.39, n s)$. The same multiple regression model predicting skill was significant $\left(F(6,30)=8.53, p<.001, R^{2}=.681\right)$, with consultation call attendance $(\beta=.45, p<$ 
$.01)$, and caseload uniquely predicting skill $(\beta=.56, p<.001)$. The multiple linear regression model was not significant in predicting acceptability $(F(6,28)=1.70, n s)$, or feasibility $(F(6,28)$ $=.28, n s)$. 
Table B1

Simple Linear Regression Analyses Predicting Knowledge Change, Skill, Acceptability, and Feasibility from Consultation Call Attendance Recommendation (80\% of calls)

\begin{tabular}{lccccc}
\hline & $B$ & $S E(B)$ & $\beta$ & $p$ & $R^{2}$ \\
\cline { 2 - 6 } Variable & & & & & \\
Knowledge change & 13.64 & 4.76 & .48 & $.01^{*}$ & .233 \\
Skill & .33 & .15 & .38 & $.03 *$ & .121 \\
Acceptability & 5.10 & 2.31 & .39 & $.04 *$ & .148 \\
Feasibility & .05 & 2.43 & .00 & .98 & .000 \\
\hline
\end{tabular}

$* p<.05, * * p<.01, * * * p<.001$ 
Table B2

Multiple Linear Regression Analysis Predicting Knowledge Change from Consultation Call Attendance Recommendation (80\% of calls) and PCIT Caseload

\begin{tabular}{lccccc}
\hline & $B$ & $S E(B)$ & $\beta$ & $p$ & $R^{2}$ \\
\cline { 2 - 6 } Model & & & & $.02^{*}$ & .272 \\
$80 \%$ calls attended & 11.20 & 5.17 & .40 & $.04^{*}$ & \\
PCIT caseload & .59 & .51 & .21 & .25 & \\
\hline
\end{tabular}

${ }^{*} p<.05$ 
Table B3

Multiple Linear Regression Analysis Predicting Skill from Consultation Call Attendance Recommendation (80\%) and PCIT Caseload

\begin{tabular}{lccccc}
\hline & $B$ & $S E(B)$ & $\beta$ & $p$ & $R^{2}$ \\
\cline { 2 - 6 } Model & & & & $.00^{* * *}$ & .616 \\
$80 \%$ calls attended & 2.94 & .94 & .39 & $.00^{* *}$ & \\
PCIT caseload & .43 & .10 & .55 & $.00^{* * *}$ & \\
\hline$* * p<.01,{ }^{* * *} p<.001$ & & & & &
\end{tabular}


Table B4

Multiple Linear Regression Analysis Predicting Skill from Consultation Call Attendance Recommendation (80\%) and Clinician Variables

\begin{tabular}{lccccc}
\hline & $B$ & $S E(B)$ & $\beta$ & $p$ & $R^{2}$ \\
\cline { 2 - 6 } Model & .02 & .04 & .05 & .67 & .68111 \\
Baseline knowledge & 3.45 & 1.04 & .45 & $.00 * *$ & \\
$80 \%$ calls attended & 1.67 & 1.03 & .21 & 1.62 & \\
Licensure & .44 & .11 & .56 & $.00 * * *$ & \\
PCIT caseload & -.50 & .46 & -.13 & .29 & \\
Therapy experience & & & & & \\
with preschool children & -.02 & .07 & -.04 & .77 & \\
Years experience & & & &
\end{tabular}




\section{Appendix C}

\section{Training to Criterion}

Cutoff scores are sometimes utilized in training outcome studies as a criterion for reaching appropriate training outcomes. In studies, a training criterion of $70-80 \%$ on scores of knowledge, skill, or adherence have been utilized in previous studies (Beidas et al., 2012; Sholomskas et al., 2005). Consistent with an approach by Beidas and colleagues (2012), a 70\% cutoff score was used in the current analyses as the population included community-based clinicians with no prior experience with the EBT. This approach has previously been used as a cutoff score when training community-based clinicians in CBT (Beidas et al., 2012).

\section{Data Analysis}

Data analyses were conducted through two simple linear regression analyses, consistent with previous research (Beidas et al., 2012). Consultation call attendance was entered into the regression model as a predictor, and the skill and knowledge training criterion variables were entered as outcomes. Neither acceptability nor feasibility criterion scores were calculated as previous research has not suggested a specific criterion for these outcomes.

\section{Descriptive Statistics}

Eighteen clinicians $(56.3 \%)$ were trained to criterion on knowledge, and seven clinicians $(21.9 \%)$ were trained to criterion on skill.

\section{Consultation Call Attendance Predicting Training to Criterion on Knowledge and Skill}

Consultation call attendance significantly predicted being trained to criterion on skill $\left(F(1,31)=5.61, \beta=.40, p<.05, R^{2}=.007\right)$, but not knowledge $(F(1,31)=.21, n s)$. 